\title{
Squarefree powers of edge ideals of forests
}

\author{
Nursel Erey \\ Department of Mathematics \\ Gebze Technical University \\ 41400 Gebze, Kocaeli, Turkey \\ nurselerey@gtu.edu.tr
}

\author{
Takayuki Hibi \\ Department of Pure and Applied Mathematics \\ Osaka University \\ Suita, Osaka 565-0871, Japan \\ hibi@math.sci.osaka-u.ac.jp
}

Submitted: Nov 26, 2020; Accepted: May 19, 2021; Published: Jun 4, 2021

(C) The authors. Released under the CC BY-ND license (International 4.0).

\begin{abstract}
Let $I(G)^{[k]}$ denote the $k$ th squarefree power of the edge ideal of $G$. When $G$ is a forest, we provide a sharp upper bound for the regularity of $I(G)^{[k]}$ in terms of the $k$-admissable matching number of $G$. For any positive integer $k$, we classify all forests $G$ such that $I(G)^{[k]}$ has linear resolution. We also give a combinatorial formula for the regularity of $I(G)^{[2]}$ for any forest $G$.
\end{abstract}

Mathematics Subject Classifications: 05E40, 13D02, 05C05

\section{Introduction}

Let $G$ be a finite simple graph with the vertex set $V(G)=\left\{x_{1}, \ldots, x_{n}\right\}$ and the edge set $E(G)$. Let $\mathbb{k}$ be a field and let $S=\mathbb{k}\left[x_{1}, \ldots, x_{n}\right]$ be the polynomial ring in $n$ variables over $\mathbb{k}$. The edge ideal of $G$, denoted by $I(G)$, is the monomial ideal generated by $x_{i} x_{j}$ such that $\left\{x_{i}, x_{j}\right\} \in E(G)$. Computation of Castelnuovo-Mumford regularity of edge ideals and their powers is a challenging problem in commutative algebra which has led to extensive literature.

Matchings in graphs appeared in the context of bounding or computing regularity. For example, it is well known that the regularity $\operatorname{reg}(I(G))$ of edge ideal of $G$ is bounded below by $\operatorname{indm}(G)+1[13]$ and above by mat $(G)+1[8]$ where $\operatorname{indm}(G)$ and $\operatorname{mat}(G)$ denote respectively the induced matching number and the matching number of the graph $G$. It is also known that such lower bound is attained when $G$ is a chordal graph [8]. These bounds were generalized to powers of edge ideals in $[2,3]$. In particular, for any positive integer $k$, the following inequalities hold:

$$
2 k+\operatorname{indm}(G)-1 \leqslant \operatorname{reg}\left(I(G)^{k}\right) \leqslant 2 k+\operatorname{mat}(G)-1 .
$$


The authors of [3] proved that the lower bound is attained when $G$ is a forest and it was conjectured in [1] that such lower bound should be also attained by chordal graphs.

In this article, we investigate squarefree powers of edge ideals. The kth squarefree power $I(G)^{[k]}$ of edge ideal of a graph $G$ is generated by the squarefree monomials in the $k$ th ordinary power $I(G)^{k}$. If $k>\operatorname{mat}(G)$, then $I(G)^{[k]}=(0)$. The study of squarefree powers was initiated in [4] and continued in [5]. Our motivation to study such powers is twofold. Firstly, thanks to the Restriction Lemma (Lemma 8) the regularity of $I(G)^{[k]}$ is bounded above by that of $I(G)^{k}$. This suggests that squarefree powers might be useful in the study of ordinary powers. For instance, if the $k$ th squarefree power does not have linear resolution, then the $k$ th ordinary power cannot have linear resolution either. The second part of our motivation comes from the fact that the generators of $I(G)^{[k]}$ correspond to the matchings in $G$ of size $k$. This makes a close connection between squarefree powers of edge ideals and the theory of matchings in graphs.

In this article, we introduce the concept of $k$-admissable matching of a graph. A matching $M$ is called $k$-admissable if there exists a partition of $M$ that satisfy certain conditions, see Definition 12. A 1-admissable matching is the same as an induced matching. Therefore, $k$-admissable matchings can be seen as generalization of induced matchings. The $k$-admissable matching number of $G$, denoted by $\operatorname{aim}(G, k)$, is the maximum size of a $k$-admissable matching. Our first main result (Theorem 25) gives an upper bound for the regularity of squarefree powers of edge ideals of forests:

Theorem 1. If $G$ is a forest, then $\operatorname{reg}\left(I(G)^{[k]}\right) \leqslant \operatorname{aim}(G, k)+k$ for every $1 \leqslant k \leqslant \operatorname{mat}(G)$.

In Theorem 28 we show that the upper bound above is attained when $k=2$ :

Theorem 2. If $G$ is a forest with $\operatorname{mat}(G) \geqslant 2$, then $\operatorname{reg}\left(I(G)^{[2]}\right)=\operatorname{aim}(G, 2)+2$.

Our second main result (Theorem 41) gives a complete classification of forests $G$ for which $I(G)^{[k]}$ has linear resolution:

Theorem 3. Let $k$ be a positive integer and let $G$ be a forest with $\operatorname{mat}(G) \geqslant k$. Then $\operatorname{reg}\left(I(G)^{[k]}\right)=2 k$ if and only if $\operatorname{aim}(G, k)=k$.

As a consequence of the above theorem, we show that for any forest $G$ and $1 \leqslant k<$ $\operatorname{mat}(G)$, if $I(G)^{[k]}$ has linear resolution, then $I(G)^{[k+1]}$ has linear resolution as well.

\section{Preliminaries}

\subsection{Definitions and notations}

Let $G$ be a finite simple graph with the vertex set $V(G)$ and the edge set $E(G)$. Given a vertex $x$ in $G$, we say $y$ is a neighbor of $x$ if $\{x, y\} \in E(G)$. We denote the set of all neighbors of $x$ by $N_{G}(x)$. We set $N_{G}[x]=N_{G}(x) \cup\{x\}$. We say the degree of $x$ in $G$ is $d$ if $x$ has exactly $d$ neighbors. A vertex of degree 0 is called an isolated vertex. A vertex of degree 1 is called a leaf. A complete graph on $n$ vertices is denoted by $K_{n}$. 
We say $H$ is a subgraph of $G$ if $V(H) \subseteq V(G)$ and $E(H) \subseteq E(G)$. A subgraph $H$ of $G$ is called an induced subgraph if for any two vertices $x, y$ in $H,\{x, y\} \in E(H)$ if and only if $\{x, y\} \in E(G)$. For any $U \subseteq V(G)$, the induced subgraph of $G$ on $U$ is the graph with the vertex set $U$ and the edge set $\{\{x, y\}: x, y \in U$ and $\{x, y\} \in E(G)\}$. For any $U \subseteq V(G)$, we denote by $G-U$ the induced subgraph of $G$ on $V(G) \backslash U$.

A graph $G$ is called connected if any two vertices of $G$ are connected by a path in $G$. A maximal connected subgraph of $G$ is called a connected component of $G$. We say $G$ is a forest if $G$ has no cycle subgraphs. A connected forest is called a tree.

A matching of $G$ is a collection of edges which are pairwise disjoint. The matching number of $G$, denoted by $\operatorname{mat}(G)$, is defined by

$$
\operatorname{mat}(G)=\max \{|M|: M \text { is a matching of } G\} .
$$

A matching $M=\left\{e_{1}, \ldots, e_{k}\right\}$ of $G$ is called an induced matching of $G$ if the induced subgraph of $G$ on $\cup_{i=1}^{k} e_{i}$ consists of the edges $e_{1}, \ldots, e_{k}$. The induced matching number of $G$, denoted by $\operatorname{indm}(G)$, is defined by

$$
\operatorname{indm}(G)=\max \{|M|: M \text { is an induced matching of } G\} .
$$

Clearly, $\operatorname{indm}(G) \leqslant \operatorname{mat}(G)$ for any graph $G$. An induced matching of size 2 is called a gap. If $\left\{e_{1}, e_{2}\right\}$ is a gap in $G$, we say the edges $e_{1}$ and $e_{2}$ form a gap in $G$. A matching $M$ of $G$ is called a perfect matching if for every vertex $x$ of $G$, there is an edge $e \in M$ such that $x \in e$.

For any positive integer $n$, we denote $\{1, \ldots, n\}$ by $[n]$.

Let $G$ be a graph with the vertex set $V(G)=\left\{x_{1}, \ldots, x_{n}\right\}$. Let $\mathbb{k}$ be a field and let $S=\mathbb{k}\left[x_{1}, \ldots, x_{n}\right]$ be the polynomial ring in $n$ variables over $\mathbb{k}$. The edge ideal of $G$, denoted by $I(G)$, is the monomial ideal defined by

$$
I(G)=\left(x_{i} x_{j}:\left\{x_{i}, x_{j}\right\} \text { is an edge of } G\right) .
$$

By abuse of notation, we will use an edge $e=\left\{x_{i}, x_{j}\right\}$ of $G$ interchangeably with the monomial $x_{i} x_{j}$. For any $1 \leqslant k \leqslant \operatorname{mat}(G)$, we define the $k$ th squarefree power of the edge ideal of $G$ by

$$
I(G)^{[k]}=\left(e_{1} \ldots e_{k}:\left\{e_{1}, \ldots, e_{k}\right\} \text { is a matching of } G\right) .
$$

We set $I(G)^{[k]}=(0)$ when $k>\operatorname{mat}(G)$. For any homogeneous ideal $I \subset S$, the (Castelnuovo-Mumford) regularity of $I$ is defined by

$$
\operatorname{reg}(I)=\max \left\{j-i: b_{i, j}(I) \neq 0\right\}
$$

where $b_{i, j}(I)$ denote the graded Betti numbers in the minimal graded free resolution of $I$. An ideal $I$ generated in degree $d$ is said to have a linear resolution if $b_{i, i+j}(I)=0$ for all $j \neq d$. 


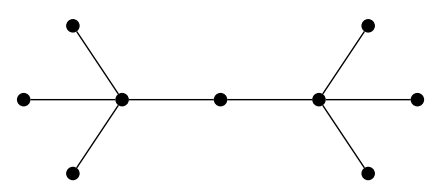

Figure 1: A graph $G$ with $\operatorname{indm}(G)=\operatorname{mat}(G)=2$.

\subsection{Background}

In this section, we collect some results that will be useful to prove our results. The following lemma shows the existence of a certain kind of leaf in forests.

Lemma 4. ([12, Proposition 4.1]) Let $T$ be a forest containing a vertex of degree at least two. Then there exists a vertex $v$ with neighbors $v_{1}, \ldots, v_{n}$ where $n \geqslant 2$ and $v_{1}, \ldots, v_{n-1}$ have degree one.

Remark 5. ([3, Remark 2.6]) Let $R=\mathbb{k}\left[x_{1}, \ldots, x_{n}\right]$ and let $I \neq R$ be a homogeneous ideal in $R$. Let $y$ be a new indeterminate and let $S=R[y]$. Then $\operatorname{reg}(I)=\operatorname{reg}(I+(y))$.

Theorem 6. ([3, Theorem 4.7]) If $G$ is a forest, then $\operatorname{reg}\left(I(G)^{k}\right)=2 k+\operatorname{indm}(G)-1$.

The authors of [4] proved the surprising result that the highest non-vanishing squarefree power of an edge ideal has linear resolution. This result will be crucial in the proof of Theorem 25.

Theorem 7. ([4, Theorem 5.1]) Let $G$ be a graph with matching number at least one. Then $I(G)^{[\operatorname{mat}(G)]}$ has linear quotients and thus it has linear resolution.

Lemma 8 (Restriction Lemma). ([9, Lemma 4.4]) Let $I \subset S$ be a monomial ideal, and let $\mathbb{F}$ be its minimal multigraded free S-resolution. Let $G(I)$ denote the minimal set of monomial generators of $I$. Furthermore, let $m$ be a monomial. We set

$$
I^{\leqslant m}=(u \in G(I) u \mid m) .
$$

Let $F_{i}=\bigoplus_{j} S\left(-\mathbf{a}_{i j}\right)$ be the ith free module in $\mathbb{F}$. Then $\mathbb{F}^{\leqslant m}$ with

$$
F_{i}^{\leqslant m}=\bigoplus_{j, \mathbf{x}^{\mathbf{a}_{i j} \mid m}} S\left(-\mathbf{a}_{i j}\right)
$$

is a subcomplex of $\mathbb{F}$ and the minimal multigraded free resolution of $I^{\leqslant m}$.

We will use the following consequence of Lemma 8:

Corollary 9. ([5, Corollary 1.3]) Let $H$ be an induced subgraph of $G$. Then $b_{i, \mathbf{a}}\left(I(H)^{[k]}\right) \leqslant$ $b_{i, \mathbf{a}}\left(I(G)^{[k]}\right)$ for all $i$ and $\mathbf{a} \in \mathbb{Z}^{n}$. In particular, $\operatorname{reg}\left(I(H)^{[k]}\right) \leqslant \operatorname{reg}\left(I(G)^{[k]}\right)$.

The following result is well-known, see for example Lemma 3.1 in the survey article [7]. 
Lemma 10. For any homogeneous ideal $I \subset S$ and any homogeneous element $m \in S$ of degree $d$ the short exact sequence

$$
0 \rightarrow \frac{S}{I: m}(-d) \rightarrow \frac{S}{I} \rightarrow \frac{S}{I+(m)} \rightarrow 0
$$

yields the following regularity bound for I:

$$
\operatorname{reg}(I) \leqslant \max \{\operatorname{reg}(I: m)+d, \operatorname{reg}(I+(m))\} .
$$

\section{$3 \quad k$-admissable matchings}

In this section, we define $k$-admissable matching of a graph, and we make some observations about their properties.

Definition 11. For any positive integers $k$ and $n$, we call a sequence $\left(a_{1}, \ldots, a_{n}\right)$ of integers a $k$-admissable sequence if the following conditions are satisfied:

1. $a_{i} \geqslant 1$ for each $i=1, \ldots, n$

2. $a_{1}+\cdots+a_{n} \leqslant n+k-1$.

Definition 12. Let $G$ be a graph with matching number mat $(G)$. Let $M$ be a matching of $G$. For any $1 \leqslant k \leqslant \operatorname{mat}(G)$ we say $M$ is $k$-admissable matching if there exists a sequence $M_{1}, \ldots, M_{r}$ of non-empty subsets of $M$ such that

1. $M=M_{1} \cup \cdots \cup M_{r}$,

2. $M_{i} \cap M_{j}=\varnothing$ for all $i \neq j$,

3. for all $i \neq j$, if $e_{i} \in M_{i}$ and $e_{j} \in M_{j}$, then $\left\{e_{i}, e_{j}\right\}$ is a gap in $G$,

4. the sequence $\left(\left|M_{1}\right|, \ldots,\left|M_{r}\right|\right)$ is $k$-admissable, and

5. the induced subgraph of $G$ on $\cup_{e \in M_{i}} e$ is a forest for all $i \in[r]$.

In such case, we say $M=M_{1} \cup \cdots \cup M_{r}$ is a $k$-admissable partition of $M$ for $G$.

Definition 13. The $k$-admissable matching number of a graph $G$, denoted by $\operatorname{aim}(G, k)$, is defined by

$$
\operatorname{aim}(G, k)=\max \{|M|: M \text { is a } k \text {-admissable matching of } G\}
$$

for $1 \leqslant k \leqslant \operatorname{mat}(G)$. We define $\operatorname{aim}(G, k)=0$ if $G$ has no $k$-admissable matching.

Remark 14. For any graph $G$, one can deduce the following properties of $k$-admissable matchings from the definition. 
1. A matching $M$ of $G$ is 1 -admissable if and only if $M$ is an induced matching of $G$. In particular, $\operatorname{indm}(G)=\operatorname{aim}(G, 1)$.

2. Let $G$ be a forest. If $M$ is a non-empty matching of $G$, then $M$ is an $|M|$-admissable matching of $G$. Therefore, $\operatorname{aim}(G, k) \geqslant k$ for every $1 \leqslant k \leqslant \operatorname{mat}(G)$.

3. If $1 \leqslant k<\operatorname{mat}(G)$ and $M$ is a $k$-admissable matching, then $M$ is $(k+1)$-admissable matching. In particular,

$$
\operatorname{indm}(G)=\operatorname{aim}(G, 1) \leqslant \operatorname{aim}(G, 2) \leqslant \ldots \leqslant \operatorname{aim}(G, \operatorname{mat}(G)) \leqslant \operatorname{mat}(G) .
$$

Moreover, if $G$ is a forest, then $\operatorname{aim}(G, \operatorname{mat}(G))=\operatorname{mat}(G)$.

4. If $H$ is an induced subgraph of $G$, then $\operatorname{aim}(H, k) \leqslant \operatorname{aim}(G, k)$ for all $k \in[\operatorname{mat}(H)]$.

Lemma 15. Let $2 \leqslant k \leqslant \operatorname{mat}(G)$. If $M$ is $k$-admissable matching, then either $M$ is $(k-1)$-admissable matching, or there exists an edge $e \in M$ such that $M \backslash\{e\}$ is $(k-1)$ admissable matching. Therefore, $\operatorname{aim}(G, k) \leqslant \operatorname{aim}(G, k-1)+1$.

Proof. Let $M=M_{1} \cup \cdots \cup M_{r}$ be a $k$-admissable partition of $M$ for $G$. Then $\left|M_{1}\right|+\cdots+$ $\left|M_{r}\right| \leqslant r+k-1$. If $\left|M_{1}\right|+\cdots+\left|M_{r}\right| \leqslant r+k-2$, then $M$ is a $(k-1)$-admissable matching. Otherwise, $\left|M_{1}\right|+\cdots+\left|M_{r}\right|=r+k-1$. Since $k \geqslant 2$, we must have $\left|M_{i}\right| \geqslant 2$ for some $i \in[r]$. Let $e \in M_{i}$. Then $M \backslash\{e\}=M_{1} \cup \cdots \cup M_{i} \backslash\{e\} \cup \cdots \cup M_{r}$ is a $(k-1)$-admissable partition of $M \backslash\{e\}$ for $G$.

Example 16. Let $G$ be the graph in Figure 2. Since $G$ has 13 vertices, $\operatorname{mat}(G) \leqslant 6$. Let $M_{1}=\{\{a, b\},\{c, d\}\}, M_{2}=\{\{f, g\},\{h, i\}\}$ and $M_{3}=\{\{j, k\},\{l, m\}\}$. Then $M=$ $M_{1} \cup M_{2} \cup M_{3}$ is a matching of size 6 and thus mat $(G)=6$. In fact, one can show that $M=M_{1} \cup M_{2} \cup M_{3}$ is a 4-admissable partition of $M$ for $G$. From Remark 14 it follows that $\operatorname{aim}(G, 4)=\operatorname{aim}(G, 5)=\operatorname{aim}(G, 6)=\operatorname{mat}(G)=6$. It is not hard to see that the induced matching number of $G$ is 3 . Therefore $\operatorname{aim}(G, 1)=3$.

Let $N_{1}=\{\{a, b\},\{c, d\}\}, N_{2}=\{\{f, g\}\}$ and $N_{3}=\{\{j, k\}\}$. Then $N=N_{1} \cup N_{2} \cup N_{3}$ is a 2 -admissable partition of $N$ for $G$. Therefore, $\operatorname{aim}(G, 2) \geqslant 4$. On the other hand, by Lemma 15 we know that $\operatorname{aim}(G, 2) \leqslant \operatorname{aim}(G, 1)+1$. Hence $\operatorname{aim}(G, 2)=4$.

Similarly, $U=M_{1} \cup M_{2} \cup N_{3}$ is a 3-admissable partition of $U$ for $G$. Therefore, $\operatorname{aim}(G, 3) \geqslant 5$. On the other hand, by Lemma 15 we know that $\operatorname{aim}(G, 3) \leqslant \operatorname{aim}(G, 2)+1$. Hence $\operatorname{aim}(G, 3)=5$.

Remark 17. If the sequence $\left(a_{1}, \ldots, a_{n}\right)$ is $k$-admissable, then so is $\left(a_{1}, \ldots, a_{n}, 1\right)$.

Lemma 18. Let $M$ be a $k$-admissable matching of a graph $G$. Then any non-empty subset of $M$ is also a $k$-admissable matching of $G$.

Proof. Let $M=M_{1} \cup \cdots \cup M_{r}$ be a $k$-admissable partition of $M$ for $G$. Then the sequence $\left(\left|M_{1}\right|, \ldots,\left|M_{r}\right|\right)$ is $k$-admissable. Therefore $|M| \leqslant r+k-1$. Let us assume that $|M|>1$ since otherwise $M$ is the only non-empty subset of itself. It suffices to show that for any $N \subseteq M$ with $|N|=|M|-1$, the matching $N$ is $k$-admissable. Without loss of generality, 
assume that $N=M \backslash\{e\}$ for some $e \in M_{1}$. If $M_{1}=\{e\}$, then $N=M_{2} \cup \cdots \cup M_{r}$ is a $k$-admissable partition of $N$ for $G$ since $|N|=|M|-1 \leqslant(r-1)+k-1$. Otherwise $N=M_{1} \backslash\{e\} \cup M_{2} \cup \cdots \cup M_{r}$ is a $k$-admissable partition of $N$ for $G$ since $|N| \leqslant r+k-1$.

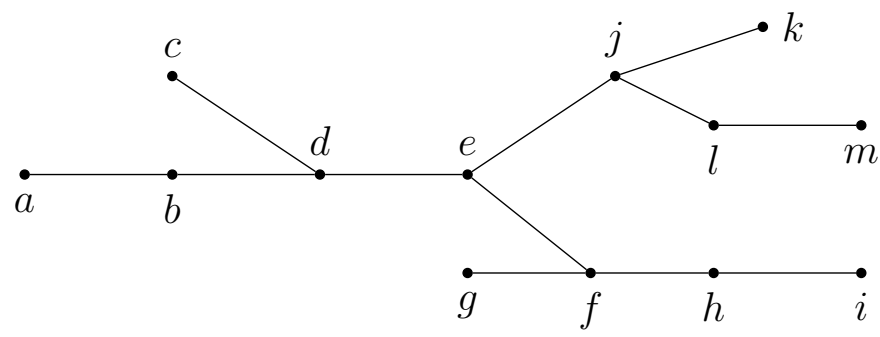

Figure 2: A graph $G$ with $\operatorname{aim}(G, 1)=3, \operatorname{aim}(G, 2)=4, \operatorname{aim}(G, 3)=5$ and $\operatorname{aim}(G, 4)=$ $\operatorname{aim}(G, 5)=\operatorname{aim}(G, 6)=\operatorname{mat}(G)=6$.

\section{Upper bounds for squarefree powers of edge ideals of forests}

In this section, we provide a sharp upper bound for $\operatorname{reg}\left(I(G)^{[k]}\right)$ where $G$ is a forest, in terms of $k$-admissable matching number of $G$. A key idea of our method is to work with a special type of vertex in a forest, which we define below.

Definition 19. Let $G$ be a forest with a leaf $x$ and its unique neighbor $y$. We say $x$ is a distant leaf if $y$ has at most one neighbor whose degree is greater than 1 . In this case, we say $\{x, y\}$ is a distant edge.

Lemma 20. Let $G$ be a forest with at least one edge. Then $G$ has a distant leaf.

Proof. If $G$ has no vertex of degree at least 2 , then $G$ consists of union of some isolated vertices and $K_{2}$ 's. In such case, every edge is a distant edge. Otherwise, the result follows from Lemma 4.

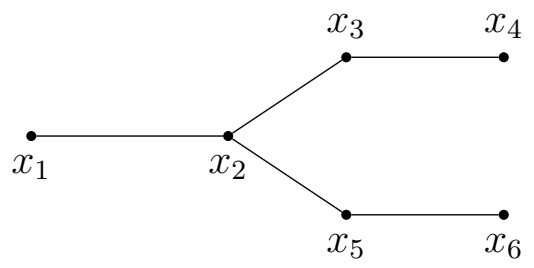

Figure 3: A tree $G$ with distant leaves $x_{4}$ and $x_{6}$.

Lemma 21. Let $G$ be a forest with a leaf $x$. Then $\operatorname{mat}(G)=\operatorname{mat}(G-\{x, y\})+1$ where $y$ is the unique neighbor of $x$. 
Proof. Any matching of $G-\{x, y\}$ can be extended to a matching of $G$ by adding the edge $\{x, y\}$. Therefore, $\operatorname{mat}(G) \geqslant \operatorname{mat}(G-\{x, y\})+1$. On the other hand, let $M=$ $\left\{e_{1}, \ldots, e_{\operatorname{mat}(G)}\right\}$ be a matching of $G$ of maximum size. If no edge of $M$ contains the vertex $y$, then $M$ is also a matching of $G-\{x, y\}$ and we get $\operatorname{mat}(G) \leqslant \operatorname{mat}(G-\{x, y\})$ as desired. Otherwise, since $M$ is a matching, there is only one edge $e_{i} \in M$ such that $y \in e_{i}$. In such case, $M \backslash\left\{e_{i}\right\}$ is a matching of $G-\{x, y\}$ and $\operatorname{mat}(G)-1 \leqslant \operatorname{mat}(G-\{x, y\})$.

Lemma 22. Let $G$ be a graph with a leaf $x$. If $y$ is the neighbor of $x$, then for all $k \geqslant 2$,

$$
I(G)^{[k]}:(x y)=I(G-\{x, y\})^{[k-1]} .
$$

Proof. If mat $(G)<k$, then $\operatorname{mat}(G-\{x, y\})<k-1$ by Lemma 21 . Then the equality is immediate as both ideals are equal to the zero ideal.

Therefore, let us assume that $2 \leqslant k \leqslant \operatorname{mat}(G)$. It is clear that $I(G-\{x, y\})^{[k-1]}$ is contained in $I(G)^{[k]}:(x y)$. To see the reverse, let $u$ be a monomial in $I(G)^{[k]}:(x y)$. Then there exists a matching $\left\{e_{1}, \ldots, e_{k}\right\}$ of $G$ such that $u x y$ is divisible by $e_{1} \ldots e_{k}$. If $x$ divides $e_{1} \ldots e_{k}$, then we may assume that $e_{1}=\{x, y\}$ since $y$ is the only neighbor of $x$. Then $u$ is divisible by $e_{2} \ldots e_{k}$ and $u \in I(G-\{x, y\})^{[k-1]}$ as $\left\{e_{2}, \ldots, e_{k}\right\}$ is a matching of $G-\{x, y\}$.

Suppose that $x$ does not divide $e_{1} \ldots e_{k}$. Then $u y$ is divisible by $e_{1} \ldots e_{k}$. Since $\left\{e_{1}, \ldots, e_{k}\right\}$ is a matching, we may assume that $y$ does not divide $e_{2} \ldots e_{k}$. Hence $u$ is divisible by $e_{2} \ldots e_{k}$ and the result follows as in the previous case.

Lemma 23. Let $G$ be a forest with matching number mat $(G) \geqslant 2$. Let $x$ be a distant leaf of $G$ with the neighbor $y$. Then for any $2 \leqslant k \leqslant \operatorname{mat}(G)$,

$$
\operatorname{aim}(G-\{x, y\}, k-1)+1 \leqslant \operatorname{aim}(G, k) .
$$

Proof. By Lemma 21 we know that the matching number of $G-\{x, y\}$ is at least $k-1$. Then by Remark 14, the forest $G-\{x, y\}$ has a $(k-1)$-admissable matching. Let $M$ be a $(k-1)$-admissable matching of $G-\{x, y\}$ of maximum cardinality. We will show that $M^{\prime}=M \cup\{\{x, y\}\}$ is a $k$-admissable matching of $G$. Let $M=M_{1} \cup \cdots \cup M_{r}$ be a $(k-1)$-admissable partition of $M$ for $G-\{x, y\}$.

If for every $e \in M$ the edges $e$ and $\{x, y\}$ form a gap in $G$, then $M^{\prime}=M_{1} \cup \cdots \cup M_{r} \cup$ $\{\{x, y\}\}$ is a $k$-admissable partition of $M^{\prime}$ for $G$ by Remark 17. So, suppose that there exists an edge $e \in M$ such that $e$ and $\{x, y\}$ do not form a gap in $G$. Since $x$ is a distant leaf of $G$, it follows that $\{\{x, y\}, f\}$ is a gap in $G$ for every $f \in M \backslash\{e\}$. Without loss of generality, suppose that $e \in M_{1}$. Let $M_{1}^{\prime}=M_{1} \cup\left\{\{x, y\}\right.$. Then $M^{\prime}=M_{1}^{\prime} \cup M_{2} \cup \cdots \cup M_{r}$ is a $k$-admissable partition of $M^{\prime}$ for $G$.

Remark 24. The lemma above is incorrect for an arbitrary leaf $x$. For example, consider the tree $G$ in Figure 3 and let $k=2$. Then $\operatorname{aim}\left(G-\left\{x_{1}, x_{2}\right\}, 1\right)=\operatorname{indm}\left(G-\left\{x_{1}, x_{2}\right\}\right)=2$ but $\operatorname{aim}(G, 2)=2$.

Theorem 25. If $G$ is a forest, then $\operatorname{reg}\left(I(G)^{[k]}\right) \leqslant \operatorname{aim}(G, k)+k$ for every $1 \leqslant k \leqslant$ $\operatorname{mat}(G)$. 
Proof. We use induction on $|V(G)|+k$. First note that if $k=1$, then the statement follows from Theorem 6 as $\operatorname{aim}(G, 1)=\operatorname{indm}(G)$. Also, if $k=\operatorname{mat}(G)$, then by Remark 14 we have $\operatorname{aim}(G, \operatorname{mat}(G))=\operatorname{mat}(G)$ and the result follows from Theorem 7 . Therefore, let us assume that $2 \leqslant k<\operatorname{mat}(G)$.

Then by Lemma 20, the forest $G$ has a distant leaf $x_{1}$ and a unique neighbor $y$. Let $x_{1}, \ldots, x_{r}$ be the neighbors of $y$ of degree 1 . We set $I_{i}=I(G)^{[k]}+\left(x_{1} y, \ldots, x_{i} y\right)$ and $G_{i}=G-\left\{x_{1}, \ldots, x_{i}\right\}$ for each $1 \leqslant i \leqslant r$. Moreover, we set $G_{0}=G$ and $I_{0}=I(G)^{[k]}$. Observe that for each $0 \leqslant i \leqslant r-1$

$$
I_{i}:\left(x_{i+1} y\right)=\left(I\left(G_{i}\right)^{[k]}:\left(x_{i+1} y\right)\right)+\left(x_{1}, \ldots, x_{i}\right)
$$

and $\left\{x_{i+1}, y\right\}$ is a distant edge of $G_{i}$. We claim that

$$
\operatorname{reg}\left(I_{i}:\left(x_{i+1} y\right)\right) \leqslant \operatorname{aim}(G, k)+k-2 \text { for all } 0 \leqslant i \leqslant r-1 .
$$

Indeed, for each $0 \leqslant i \leqslant r-1$, $\operatorname{since} \operatorname{mat}\left(G_{i}\right)=\operatorname{mat}(G)$ and $\operatorname{mat}\left(G_{i}-\left\{x_{i+1}, y\right\}\right) \geqslant k-1$, we obtain

$$
\begin{array}{rlr}
\operatorname{reg}\left(I_{i}:\left(x_{i+1} y\right)\right) & =\operatorname{reg}\left(I\left(G_{i}\right)^{[k]}:\left(x_{i+1} y\right)\right) & \text { (Remark 5) } \\
& =\operatorname{reg}\left(I\left(G_{i}-\left\{x_{i+1}, y\right\}\right)^{[k-1]}\right) & \text { (Lemma 22) } \\
& \leqslant \operatorname{aim}\left(G_{i}-\left\{x_{i+1}, y\right\}, k-1\right)+k-1 & \text { (induction assumption) } \\
& \leqslant \operatorname{aim}\left(G_{i}, k\right)+k-2 & \text { (Lemma 23) } \\
& \leqslant \operatorname{aim}(G, k)+k-2 & ((4) \text { of Remark 14) }
\end{array}
$$

which proves Eq. (1). We can apply Eq. (1) and Lemma 10 successively to eliminate $x_{1}$ and its duplicates as follows.

$$
\begin{aligned}
\operatorname{reg}\left(I(G)^{[k]}\right) & \leqslant \max \left\{\operatorname{reg}\left(I(G)^{[k]}:\left(x_{1} y\right)\right)+2, \operatorname{reg}\left(I_{1}\right)\right\} \\
& \leqslant \max \left\{\operatorname{aim}(G, k)+k, \operatorname{reg}\left(I_{1}:\left(x_{2} y\right)\right)+2, \operatorname{reg}\left(I_{2}\right)\right\} \\
& \leqslant \max \left\{\operatorname{aim}(G, k)+k, \operatorname{reg}\left(I_{2}:\left(x_{3} y\right)\right)+2, \operatorname{reg}\left(I_{3}\right)\right\} \\
& \leqslant \vdots \\
& \leqslant \max \left\{\operatorname{aim}(G, k)+k, \operatorname{reg}\left(I_{r}\right)\right\} .
\end{aligned}
$$

Therefore, it suffices to show that $\operatorname{reg}\left(I_{r}\right) \leqslant \operatorname{aim}(G, k)+k$. By Lemma 10 we have

$$
\operatorname{reg}\left(I_{r}\right) \leqslant \max \left\{\operatorname{reg}\left(I_{r}:(y)\right)+1, \operatorname{reg}\left(I_{r}+(y)\right)\right\}
$$

Then it suffices to show that the maximum in the above inequality is at most $\operatorname{aim}(G, k)+k$. Note that by Lemma 21 we have

$$
\operatorname{mat}(G-\{y\})=\operatorname{mat}\left(G-\left\{x_{1}, y\right\}\right)=\operatorname{mat}(G)-1 \geqslant k .
$$

Remark 5, induction assumption and (4) of Remark 14 imply

$$
\operatorname{reg}\left(I_{r}+(y)\right)=\operatorname{reg}\left(I(G-\{y\})^{[k]}\right) \leqslant \operatorname{aim}(G-\{y\}, k)+k \leqslant \operatorname{aim}(G, k)+k .
$$


Since $x_{1}$ is a distant leaf of $G$, either $N_{G}(y)=\left\{x_{1}, \ldots, x_{r}\right\}$ or $N_{G}(y)=\left\{x_{1}, \ldots, x_{r}, z\right\}$ for some vertex $z$ of degree greater than 1 . We will consider these cases separately.

Case 1: Suppose that $N_{G}(y)=\left\{x_{1}, \ldots, x_{r}\right\}$. Then the induced subgraph of $G$ on $N_{G}[y]$ is a connected component of $G$ and $\operatorname{aim}(G, k) \geqslant \operatorname{aim}\left(G_{r}, k\right)+1$ by Remark 17 . Since $I_{r}:(y)=I\left(G_{r}\right)^{[k]}+\left(x_{1}, \ldots, x_{r}\right)$, by Remark 5 and induction assumption we get

$$
\operatorname{reg}\left(I_{r}:(y)\right)=\operatorname{reg}\left(I\left(G_{r}\right)^{[k]}\right) \leqslant \operatorname{aim}\left(G_{r}, k\right)+k \leqslant \operatorname{aim}(G, k)+k-1 .
$$

Case 2: Suppose that $N_{G}(y)=\left\{x_{1}, \ldots, x_{r}, z\right\}$ for some vertex $z$ of degree greater than 1. Observe that $I_{r}:(y)=z I(G-\{y, z\})^{[k-1]}+I(G-\{y, z\})^{[k]}+\left(x_{1}, \ldots, x_{r}\right)$. By Lemma 10

$$
\operatorname{reg}\left(I_{r}:(y)\right) \leqslant \max \left\{\operatorname{reg}\left(\left(I_{r}:(y)\right):(z)\right)+1, \operatorname{reg}\left(\left(I_{r}:(y)\right)+(z)\right)\right\} .
$$

We will now show that the maximum in (2) is at most aim $(G, k)+k-1$ which will complete the proof. Observe that by Remark 5 we have

$$
\operatorname{reg}\left(\left(I_{r}:(y)\right):(z)\right)=\operatorname{reg}\left(I(G-\{y, z\})^{[k-1]}\right)=\operatorname{reg}\left(I\left(G-\left\{x_{1}, y, z\right\}\right)^{[k-1]}\right) .
$$

Applying respectively Remark 5, Corollary 9, induction assumption on $G-\left\{x_{1}, y\right\}$ and Lemma 23, we obtain

$$
\begin{aligned}
\operatorname{reg}\left(\left(I_{r}:(y)\right):(z)\right) & \leqslant \operatorname{reg}\left(I\left(G-\left\{x_{1}, y\right\}\right)^{[k-1]}\right) \\
& \leqslant \operatorname{aim}\left(G-\left\{x_{1}, y\right\}, k-1\right)+k-1 \\
& \leqslant \operatorname{aim}(G, k)+k-2 .
\end{aligned}
$$

Observe that Remark 5 implies $\operatorname{reg}\left(\left(I_{r}:(y)\right)+(z)\right)=\operatorname{reg}\left(I(G-\{y, z\})^{[k]}\right)$. We may assume that the matching number of $G-\{y, z\}$ is at least $k$ since otherwise the proof is immediate. By induction, we have

$$
\operatorname{reg}\left(I(G-\{y, z\})^{[k]}\right) \leqslant \operatorname{aim}(G-\{y, z\}, k)+k .
$$

Therefore, it remains to show that $\operatorname{aim}(G-\{y, z\}, k)+1 \leqslant \operatorname{aim}(G, k)$. Indeed, keeping Remark 17 in mind, any $k$-admissable matching of $G-\{y, z\}$ can be extended to a $k$ admissable matching of $G$ by adding the edge $\left\{x_{1}, y\right\}$.

\subsection{Second squarefree powers}

The goal of this section is to show that the upper bound in Theroem 25 is attained when $k=2$. To this end, we will show that Betti numbers do not vanish in certain degrees. The following fact was established in the proof of [5, Theorem 2.1].

Lemma 26. If $M$ is a 1-admissable matching of $G$ which is also a perfect matching, then for all $1 \leqslant k \leqslant|M|$

$$
b_{|M|-k+1,2|M|}\left(S / I(G)^{[k]}\right) \neq 0 .
$$


We now extend Lemma 26 to 2-admissable matchings as follows:

Lemma 27. If $M$ is a 2-admissable perfect matching of $G$, then for all $2 \leqslant k \leqslant|M|$

$$
b_{|M|-k+1,2|M|}\left(S / I(G)^{[k]}\right) \neq 0 .
$$

Proof. We may assume that $\operatorname{mat}(G) \geqslant 2$ as the statement is vacuously true otherwise. If $M$ is 1-admissable matching, then the result follows from Lemma 26.

So, let us assume that $M$ is not 1-admissable. Let

$$
M=\left\{\left\{x_{1}, x_{2}\right\},\left\{x_{3}, x_{4}\right\}, \ldots,\left\{x_{2 r-1}, x_{2 r}\right\}\right\}
$$

so that $|M|=r$. Since $M$ is not an induced matching of $G$, without loss of generality, we may assume that $\left\{x_{2}, x_{3}\right\}$ is an edge of $G$.

Claim: $G$ has exactly $r+1$ edges.

Proof of the claim: Let $M=M_{1} \cup \cdots \cup M_{q}$ be a 2-admissable partition of $M$ for $G$. By condition (3) of Definition 12 we may assume that both $\left\{x_{1}, x_{2}\right\}$ and $\left\{x_{3}, x_{4}\right\}$ are in $M_{1}$. Since the sequence $\left(\left|M_{1}\right|, \ldots,\left|M_{q}\right|\right)$ is 2-admissable, we have $\left|M_{1}\right|+\cdots+\left|M_{q}\right| \leqslant q+1$. On the other hand, since $\left|M_{1}\right| \geqslant 2$ and $\left|M_{i}\right| \geqslant 1$ for all $i \geqslant 2$, we obtain $\left|M_{1}\right|=2$ and $\left|M_{i}\right|=1$ for each $i \geqslant 2$. The claim then follows from conditions (3) and (5) of Definition 12 together with the fact that $M$ is a perfect matching of $G$.

Having proved our claim, we can now write

$$
I(G)=\left(x_{1} x_{2}, x_{2} x_{3}, x_{3} x_{4}, x_{5} x_{6}, \ldots, x_{2 r-1} x_{2 r}\right) .
$$

By Lemma 22 we set $J:=I(G)^{[k]}:\left(x_{1} x_{2}\right)=I\left(G-\left\{x_{1}, x_{2}\right\}\right)^{[k-1]}$. Also we set $K=$ $I(G)^{[k]}+\left(x_{1} x_{2}\right)$. The short exact sequence

$$
0 \rightarrow \frac{S}{J}(-2) \rightarrow \frac{S}{I(G)^{[k]}} \rightarrow \frac{S}{K} \rightarrow 0
$$

yields the long exact sequence

$$
\cdots \rightarrow \operatorname{Tor}_{r-k+2}(S / K, \mathbb{k})_{2 r} \rightarrow \operatorname{Tor}_{r-k+1}((S / J)(-2), \mathbb{k})_{2 r} \rightarrow \operatorname{Tor}_{r-k+1}\left(S / I(G)^{[k]}, \mathbb{k}\right)_{2 r} \rightarrow \cdots
$$

Since $M \backslash\left\{\left\{x_{1}, x_{2}\right\}\right\}$ is perfect induced matching of $G-\left\{x_{1}, x_{2}\right\}$, by Lemma 26 we have $\operatorname{Tor}_{r-k+1}((S / J)(-2), \mathbb{k})_{2 r} \neq 0$. Therefore, it suffices to show that $\operatorname{Tor}_{r-k+2}(S / K, \mathbb{k})_{2 r}=0$. The short exact sequence

$$
0 \rightarrow \frac{S}{K:\left(x_{2}\right)}(-1) \rightarrow \frac{S}{K} \rightarrow \frac{S}{K+\left(x_{2}\right)} \rightarrow 0
$$

yields the long exact sequence

$$
\begin{aligned}
& \cdots \rightarrow \operatorname{Tor}_{r-k+2}\left(\left(S /\left(K:\left(x_{2}\right)\right)\right)(-1), \mathbb{k}\right)_{2 r} \rightarrow \\
& \operatorname{Tor}_{r-k+2}(S / K, \mathbb{k})_{2 r} \rightarrow \operatorname{Tor}_{r-k+2}\left(S /\left(K+\left(x_{2}\right)\right), \mathbb{k}\right)_{2 r} \rightarrow \cdots
\end{aligned}
$$


We will now use the fact that all non-zero Betti numbers of a squarefree monomial ideal lie in squarefree multidegrees. Observe that the squarefree monomial ideal $K+\left(x_{2}\right)$ lies in a polynomial ring with less than $2 r$ variables, more precisely, $K+\left(x_{2}\right) \subseteq \mathbb{k}\left[x_{2}, \ldots, x_{2 r}\right]$. Then $\operatorname{Tor}_{i}\left(\left(S /\left(K+\left(x_{2}\right)\right)\right), \mathbb{k}\right)_{2 r}=0$ for every $i$. On the other hand, observe that

$$
K:\left(x_{2}\right)=\left(I(G)^{[k]}+\left(x_{1} x_{2}\right)\right):\left(x_{2}\right)=x_{3} I\left(G-\left\{x_{2}, x_{3}\right\}\right)^{[k-1]}+I\left(G-\left\{x_{2}, x_{3}\right\}\right)^{[k]}+\left(x_{1}\right) .
$$

Then the squarefree monomial ideal $K:\left(x_{2}\right)$ lies in a polynomial ring with $2 r-2$ variables because the variables $x_{2}$ and $x_{4}$ have disappeared. Therefore $\operatorname{Tor}_{r-k+2}(S /(K$ : $\left.\left.\left(x_{2}\right)\right), \mathbb{k}\right)_{2 r-1}=0$ or, equivalently $\operatorname{Tor}_{r-k+2}\left(\left(S /\left(K:\left(x_{2}\right)\right)\right)(-1), \mathbb{k}\right)_{2 r}=0$.

We now give a formula for the regularity of $I(G)^{[2]}$ when $G$ is a forest.

Theorem 28. If $G$ is a forest with $\operatorname{mat}(G) \geqslant 2$, then $\operatorname{reg}\left(I(G)^{[2]}\right)=\operatorname{aim}(G, 2)+2$.

Proof. By Theorem 25 and Corollary 9 it suffices to show that for every 2-admissable matching $M$ of $G$, the inequality $\operatorname{reg}\left(I(H)^{[2]}\right) \geqslant|M|+2$ holds where $H$ is the induced subgraph of $G$ on $\cup_{e \in M} e$. Note that $M$ is a perfect matching and 2-admissable matching of $H$. Then by Lemma 27 we get

$$
b_{|M|-2,2|M|}\left(I(H)^{[2]}\right)=b_{|M|-1,2|M|}\left(S / I(H)^{[2]}\right) \neq 0
$$

and thus $\operatorname{reg}\left(I(H)^{[2]}\right) \geqslant|M|+2$ as desired.

In particular, Theorem 28 gives a lower bound for the regularity of second squarefree power of edge ideal of any graph.

Corollary 29. If $G$ is a graph with $\operatorname{mat}(G) \geqslant 2$, then $\operatorname{reg}\left(I(G)^{[2]}\right) \geqslant \operatorname{aim}(G, 2)+2$.

Proof. Follows from Corollary 9.

A graph $G$ that satisfies $\operatorname{indm}(G)=\operatorname{mat}(G)$ is called a Cameron-Walker graph. Such graphs were studied from a commutative algebra point of view in [11]. The following proposition shows that the upper bound in Theorem 25 is sharp.

Proposition 30. If $G$ is a Cameron-Walker forest, then for all $1 \leqslant k \leqslant \operatorname{mat}(G)$, $\operatorname{reg}\left(I(G)^{[k]}\right)=\operatorname{aim}(G, k)+k$.

Proof. By Remark 14 it follows that $\operatorname{aim}(G, k)=\operatorname{indm}(G)$ for all $1 \leqslant k \leqslant \operatorname{mat}(G)$. By Theorem 25 we only need to show that $\operatorname{reg}\left(I(G)^{[k]}\right) \geqslant \operatorname{indm}(G)+k$. Let $M$ be an induced matching of $G$ of maximum cardinality. Let $H$ be the induced subgraph of $G$ on $\cup_{e \in M} e$. Then $M$ is a perfect matching of $H$. The result then follows from Lemma 26 and Corollary 9.

Using the structural classification of Cameron-Walker graphs [11], for any given positive integer $m$, one can construct a Cameron-Walker tree $G$ with $\operatorname{indm}(G)=\operatorname{mat}(G)=m$. Figure 1 illustrates an example with $m=2$.

Based on the results of this section and Macaulay2 [6] computations, we expect that the upper bound in Theorem 25 would give the exact formula for the regularity of squarefree powers of edge ideals of forests. Thus, we propose the following conjecture.

Conjecture 31. If $G$ is a forest, then $\operatorname{reg}\left(I(G)^{[k]}\right)=\operatorname{aim}(G, k)+k$ for every $1 \leqslant k \leqslant$ $\operatorname{mat}(G)$. 


\section{Characterization of squarefree powers with linear resolutions}

In this section, we will classify forests $G$ such that $I(G)^{[k]}$ has linear resolution. From Theorem 6 it follows that $I(G)^{k}$ has linear resolution if and only if $\operatorname{indm}(G)=1$ when $G$ is a forest. So, for ordinary powers, such characterization does not depend on $k$, and the class of forests with induced matching number equal to one is rather small. On the other hand, we will see that linearity of resolution of $I(G)^{[k]}$ depends on both the forest $G$ and the integer $k$.

Let us briefly recall some definitions about simplicial complexes. A simplicial complex $\Delta$ on a finite vertex set $V(\Delta)$ is a collection of subsets of $V(\Delta)$ such that if $F \in \Delta$, then every subset of $F$ is also in $\Delta$. Each element of $\Delta$ is called a face of $\Delta$. If $F$ is a maximal face of $\Delta$ with respect to inclusion, then we say $F$ is a facet of $\Delta$. We write $\Delta=\left\langle F_{1}, \ldots, F_{r}\right\rangle$ if $F_{1}, \ldots, F_{r}$ are all the facets of $\Delta$. We say $\Delta$ is connected if for every pair of vertices $u$ and $v$ there exists a sequence $F_{1}, \ldots, F_{s}$ of facets of $\Delta$ such that $u \in F_{1}$, $v \in F_{s}$ and $F_{i} \cap F_{i+1} \neq \varnothing$ for each $i=1, \ldots, s-1$.

Definition 32. Let $I \subseteq S=\mathbb{k}\left[x_{1}, \ldots, x_{n}\right]$ be a monomial ideal and let $\alpha=\left(\alpha_{1}, \ldots, \alpha_{n}\right) \in$ $\mathbb{N}^{n}$ be a multidegree. The upper-Koszul simplicial complex associated with $I$ at degree $\alpha$, denoted by $K^{\alpha}(I)$, is the simplicial complex over $V=\left\{x_{1}, \ldots, x_{n}\right\}$ whose faces are:

$$
\left\{W \subseteq V \mid \frac{x_{1}^{\alpha_{1}} \ldots x_{n}^{\alpha_{n}}}{\prod_{u \in W} u} \in I\right\}
$$

Hochster's formula ([14, Theorem 1.34]) describe multigraded Betti numbers of a monomial ideal $I$ in terms of reduced homology groups of upper-Koszul simplicial complexes as follows:

$$
b_{i, \alpha}(I)=\operatorname{dim}_{\mathbb{k}} \tilde{H}_{i-1}\left(K^{\alpha}(I) ; \mathbb{k}\right) \quad \text { for } i \geqslant 0 \text { and } \alpha \in \mathbb{N}^{n} .
$$

Notation 33. Let $m=x_{1}^{\alpha_{1}} \ldots x_{n}^{\alpha_{n}}$ be a monomial in $\mathbb{k}\left[x_{1}, \ldots, x_{n}\right]$. To ease the notation, the monomial $m$ and the multidegree $\left(\alpha_{1}, \ldots, \alpha_{n}\right)$ will be used interchangeably. Moreover, if $m=x_{i_{1}} \ldots x_{i_{k}}$ is squarefree, we will denote the set $\left\{x_{i_{1}}, \ldots, x_{i_{k}}\right\}$ by $m$.

Lemma 34. Let $I$ be a squarefree monomial ideal minimally generated by $m_{1}, \ldots, m_{t}$. Let $m=\operatorname{lcm}\left(m_{1}, \ldots, m_{t}\right)$. Then $K^{m}(I)=\left\langle m / m_{1}, \ldots, m / m_{t}\right\rangle$.

Proof. By definition of the upper-Koszul simplicial complex, it is clear that each $\mathrm{m} / \mathrm{m}_{i}$ corresponds to a face of $K^{m}(I)$. Moreover, $m / m_{i}$ corresponds to a maximal face since $m_{i}$ is a minimal monomial generator. Lastly, if $u$ is a monomial that corresponds to a face of $K^{m}(I)$, then $m / u \in I$. Then there is a monomial $v$ such that $m / u=v m_{i}$ for some $i \in[t]$. This implies that the face $u$ is contained in the facet $m / m_{i}$.

The following lemma is well-known in graph theory.

Lemma 35. Let $G$ be a graph with connected components $G_{1}, \ldots, G_{r}$. Then $G$ has a perfect matching if and only if $G_{i}$ has a perfect matching for each $i \in[r]$. 
Lemma 36. Let $G$ be a graph which has a perfect matching. Then for any vertex $x$ of $G$, the graph $G-\{x\}$ has no perfect matching.

Proof. If a graph has perfect matching, then it has even number of vertices.

Lemma 37. Let $G$ be a graph with connected components $G_{1}, \ldots, G_{r}$ where $r \geqslant 2$. Suppose that $G$ has a perfect matching. If $x \in V\left(G_{1}\right)$ and $y \in V\left(G_{2}\right)$, then $G-\{x, y\}$ has no perfect matching.

Proof. By Lemma 35 each $G_{i}$ has a perfect matching. Let $U_{1}, \ldots, U_{t}$ be the connected components of $G_{1}-\{x\}$ and $V_{1}, \ldots, V_{s}$ be the connected components of $G_{2}-\{y\}$. Then the connected components of $G-\{x, y\}$ are $U_{1}, \ldots, U_{t}, V_{1}, \ldots, V_{s}, G_{3}, \ldots, G_{r}$. By Lemma 36 the graph $G_{1}-\{x\}$ has no perfect matching. Then by Lemma 35 there exists $U_{j}$ which has no perfect matching. Since $U_{j}$ is also a connected component of $G-\{x, y\}$, it follows that $G-\{x, y\}$ has no perfect matching.

Notation 38. If $M=\left\{e_{1}, \ldots, e_{k}\right\}$ is a matching of $G$, then we will write $u_{M}$ for the squarefree monomial $e_{1} \ldots e_{k}=\prod_{\substack{x_{i} \in e, e \in M}} x_{i}$.

Lemma 39. Let $G$ be a graph with a $k$-admissable perfect matching $M$. Let $M=M_{1} \cup$ $\cdots \cup M_{r}$ be a $k$-admissable partition of $M$ for $G$. Using Notation 38 let $x \mid u_{M_{i}}$ and $y \mid u_{M_{j}}$ for some vertices $x$ and $y$ with $i \neq j$. Then $x$ and $y$ are in different connected components of $G$.

Proof. If $\{a, b\}$ is an edge of $G$, then since $M$ is a perfect matching, $a \mid u_{M_{p}}$ and $b \mid u_{M_{q}}$ for some $p$ and $q$. Since $M$ is $k$-admissable, we get $p=q$. Therefore there is no path in $G$ that connects $x$ and $y$.

We use Notation 38 again to state the next lemma:

Lemma 40. Let $H$ be a graph with a $k$-admissable perfect matching $M$ of cardinality $k+1$. Then the simplicial complex $K^{u_{M}}\left(I(H)^{[k]}\right)$ is disconnected.

Proof. Let $V(H)=\left\{x_{1}, \ldots, x_{2 k+2}\right\}$. Then $u_{M}=x_{1} \ldots x_{2 k+2}$ and $u_{M}$ is the least common multiple of minimal monomial generators of $I(H)^{[k]}$. By Lemma 34, observe that every facet of $K^{u_{M}}\left(I(H)^{[k]}\right)$ consists of 2 vertices. In fact, $\left\{x_{i}, x_{j}\right\}$ is a facet of $K^{u_{M}}\left(I(H)^{[k]}\right)$ if and only if $H-\left\{x_{i}, x_{j}\right\}$ has a perfect matching. To see this, let $\mathcal{F}$ be the set of facets of $K^{u_{M}}\left(I(H)^{[k]}\right)$. Then by Lemma 34

$$
\begin{aligned}
\left\{x_{i}, x_{j}\right\} \in \mathcal{F} & \Longleftrightarrow x_{i} x_{j}=\frac{u_{M}}{u_{N}} \text { for some matching } N \text { of } H \text { of size } k \\
& \Longleftrightarrow x_{i} x_{j}=\frac{u_{M}}{u_{N}} \text { for some matching } N \text { of } H-\left\{x_{i}, x_{j}\right\} \text { of size } k \\
& \Longleftrightarrow u_{N}=V(H) \backslash\left\{x_{i}, x_{j}\right\} \text { for some matching } N \text { of } H-\left\{x_{i}, x_{j}\right\} \text { of size } k \\
& \Longleftrightarrow H-\left\{x_{i}, x_{j}\right\} \text { has a perfect matching. }
\end{aligned}
$$


Let $M=M_{1} \cup M_{2} \cup \cdots \cup M_{r}$ be a $k$-admissable partition of $M$ for $H$. Then by definition of $k$-admissable sequence, we must have $r \geqslant 2$. Let $e_{1} \in M_{1}$ and $e_{2} \in M_{2}$. Then both $e_{1}$ and $e_{2}$ are facets of $K^{u_{M}}\left(I(H)^{[k]}\right)$. We claim that there is no sequence of faces that connects a vertex of $e_{1}$ to a vertex of $e_{2}$. To this end, we will show that if $\left\{x_{i}, x_{j}\right\}$ is a facet of $K^{u_{M}}\left(I(H)^{[k]}\right)$, then $x_{i} x_{j} \mid u_{M_{q}}$ for some $q \in[r]$. Assume for a contradiction there is a facet $\left\{x_{i}, x_{j}\right\}$ such that $x_{i} \mid u_{M_{i^{\prime}}}$ and $x_{j} \mid u_{M_{j^{\prime}}}$ for some $i^{\prime} \neq j^{\prime}$. Then by Lemma 39 the vertices $x_{i}$ and $x_{j}$ belong to different connected components of $H$. Then by Lemma 37, $H-\left\{x_{i}, x_{j}\right\}$ has no perfect matching, which is a contradiction.

The authors of [5] classified all forests $G$ such that $I(G)^{[2]}$ has linear resolution, see [5, Theorem 5.3]. Our next theorem solves this classification problem for any squarefree power.

Theorem 41. Let $k \geqslant 1$ be an integer and let $G$ be a forest with $\operatorname{mat}(G) \geqslant k$. Then $\operatorname{reg}\left(I(G)^{[k]}\right)=2 k$ if and only if $\operatorname{aim}(G, k)=k$.

Proof. If $\operatorname{aim}(G, k)=k$, then by Theorem 25 it follows that $\operatorname{reg}\left(I(G)^{[k]}\right)=2 k$. Suppose that $\operatorname{aim}(G, k) \neq k$. Then $\operatorname{aim}(G, k)>k$ by Remark 14 . Let $N$ be a $k$-admissable matching of $G$ of cardinality $\operatorname{aim}(G, k)$. Then by Lemma 18 there exists a $k$-admissable matching $M$ of $G$ which has $k+1$ elements. Let $H$ be the induced subgraph of $G$ on $\cup_{e \in M}$ e. Then $M$ is a perfect matching of $H$. By Corollary 9 we get $\operatorname{reg}\left(I(G)^{[k]}\right) \geqslant$ $\operatorname{reg}\left(I(H)^{[k]}\right)$. By Lemma 40, the simplicial complex $K^{u_{M}}\left(I(H)^{[k]}\right)$ is disconnected. Then $\operatorname{dim}_{\mathbb{k}} \tilde{H}_{0}\left(K^{u_{M}}\left(I(H)^{[k]}\right) ; \mathbb{k}\right)>0$. From the Hochster's formula, we get $b_{1,2 k+2}\left(I(H)^{[k]}\right) \neq 0$ and thus $\operatorname{reg}\left(I(H)^{[k]}\right) \geqslant 2 k+1$.

Herzog, Hibi and Zheng [10] proved that if an edge ideal $I(G)$ has linear resolution, then $I(G)^{k}$ has linear resolution for all $k \geqslant 1$. It is an open problem to determine for a given integer $k$, whether linearity of resolution of $I(G)^{k}$ implies the same property for $I(G)^{k+1}$. Relevantly, Theorem 41 has an interesting consequence regarding linear resolutions of consecutive squarefree powers:

Corollary 42. Let $G$ be a forest and $1 \leqslant k<\operatorname{mat}(G)$. If $I(G)^{[k]}$ has linear resolution, then $I(G)^{[k+1]}$ has linear resolution.

Proof. Suppose that $\operatorname{reg}\left(I(G)^{[k]}\right)=2 k$. Then by Theorem 41 we get $\operatorname{aim}(G, k)=k$. Now, observe that

$$
\begin{aligned}
\operatorname{reg}\left(I(G)^{[k+1]}\right) & \leqslant \operatorname{aim}(G, k+1)+k+1 \quad(\text { Theorem 25) } \\
& \leqslant \operatorname{aim}(G, k)+1+k+1 \quad \text { (Lemma 15) } \\
& =2 k+2
\end{aligned}
$$

and thus $I(G)^{[k+1]}$ has linear resolution.

\section{Acknowledgements}

We thank the anonymous referee for her/his careful reading of the paper and helpful comments. 


\section{References}

[1] A. Banerjee, S. K. Beyarslan, H. T. Hà, Regularity of edge ideals and their powers, Advances in algebra, 17-52, Springer Proc. Math. Stat., 277, Springer, Cham, 2019.

[2] A. Banerjee, S. K. Beyarslan, H. T. Hà, Regularity of powers of edge ideals: from local properties to global bounds, Algebr. Comb. 3 (2020), no. 4, 839-854.

[3] S. Beyarslan, H. T. Hà, T.N. Trung, Regularity of powers of forests and cycles, J. Algebraic Combin., 42(4):1077-1095, 2015.

[4] M. Bigdeli, J. Herzog, R. Zaare-Nahandi, On the index of powers of edge ideals, Comm. Algebra 46 (3) (2018), 1080-1095.

[5] N. Erey, J. Herzog, T. Hibi, S. Saeedi Madani, Matchings and squarefree powers of edge ideals, arXiv:1909.11420, 2019.

[6] D. R. Grayson, M. E. Stillman, Macaulay2, a software system for research in algebraic geometry, Available at http://www.math.uiuc.edu/Macaulay2/.

[7] H. T. Há, Regularity of squarefree monomial ideals, Connections between algebra, combinatorics, and geometry, 251-276, Springer Proc. Math. Stat., 76, Springer, New York, 2014.

[8] H. T. Há, A. Van Tuyl, Monomial ideals, edge ideals of hypergraphs, and their graded Betti numbers, J. Algebraic Combin. 27 (2008), no. 2, 215-245.

[9] J. Herzog, T. Hibi, X. Zheng, Dirac's theorem on chordal graphs and Alexander duality, European J. Combin., 25 (2004), 949-960.

[10] J. Herzog, T. Hibi, X. Zheng, Monomial ideals whose powers have a linear resolution, Math. Scand., 95 (2004), 23-32.

[11] T. Hibi, A. Higashitani, K. Kimura, A. O'Keefe, Algebraic study on Cameron-Walker graphs, J. Algebra 422 (2015), 257-269.

[12] S. Jacques, M. Katzman, The Betti numbers of forests, arXiv:math/0501226, 2005.

[13] M. Katzman, Characteristic-independence of Betti numbers of graph ideals, J. Combin. Theory, Ser. A, 113:435-454, 2006.

[14] E. Miller, B. Sturmfels, Combinatorial Commutative Algebra, GTM, vol. 227. Springer, Berlin (2004). 